Götz, Christina; Kutzelmann, Sabine; Massler, Ute

Mehrsprachiges Lesetheater: Ergebnisse zur Akzeptanz dieser neuen mehrsprachigen Lesefördermethode

Mehrsprachiges Lesetheater. Handbuch zu Theorie und Praxis. Opladen; Berlin; Toronto : Verlag Barbara Budrich 2017, S. 43-56

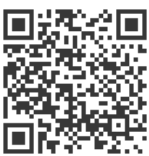

Quellenangabe/ Reference:

Götz, Christina; Kutzelmann, Sabine; Massler, Ute: Mehrsprachiges Lesetheater: Ergebnisse zur

Akzeptanz dieser neuen mehrsprachigen Lesefördermethode - In: Mehrsprachiges Lesetheater.

Handbuch zu Theorie und Praxis. Opladen; Berlin; Toronto : Verlag Barbara Budrich 2017, S. 43-56 -

URN: urn:nbn:de:0111-pedocs-216634 - DOI: 10.25656/01:21663

https://nbn-resolving.org/urn:nbn:de:0111-pedocs-216634

https://doi.org/10.25656/01:21663

in Kooperation mit / in cooperation with:

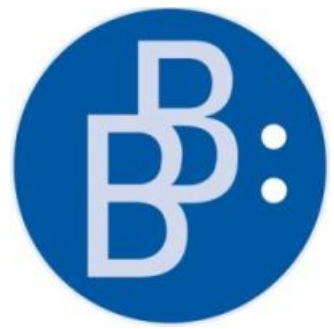

https://www.budrich.de

\section{Nutzungsbedingungen}

Gewährt wird ein nicht exklusives, nicht übertragbares, persönliches und beschränktes Recht auf Nutzung dieses Dokuments. Dieses Dokument ist ausschließlich für den persönlichen, nicht-kommerziellen Gebrauch bestimmt. Die Nutzung stellt keine Übertragung des Eigentumsrechts an diesem Dokument dar und gilt vorbehaltlich der folgenden Einschränkungen: Auf sämtlichen Kopien dieses Dokuments müssen alle Urheberrechtshinweise und sonstigen Hinweise auf gesetzlichen Schutz beibehalten werden. Sie dürfen dieses Dokument nicht in irgendeiner Weise abändern, noch dürfen Sie dieses Dokument für öffentliche oder kommerzielle Zwecke vervielfältigen, öffentlich ausstellen, aufführen, vertreiben oder anderweitig nutzen.

Mit der Verwendung dieses Dokuments erkennen Sie die Nutzungsbedingungen an.

\section{Kontakt / Contact:}

\section{peDOCS}

DIPF | Leibniz-Institut für Bildungsforschung und Bildungsinformation Informationszentrum (IZ) Bildung

E-Mail: pedocs@dipf.de

Internet: www.pedocs.de

\section{Terms of use}

We grant a non-exclusive, non-transferable, individual and limited right to using this document.

This document is solely intended for your personal, non-commercial use. Use of this document does not include any transfer of property rights and it is conditional to the following limitations: All of the copies of this documents must retain all copyright information and other information regarding legal protection. You are not allowed to alter this document in any way, to copy it for public or commercial purposes, to exhibit the document in public, to perform, distribute or otherwise use the document in public.

By using this particular document, you accept the above-stated conditions of use. 
Sabine Kutzelmann Ute Massler Klaus Peter Kristina Götz Angelika Ilg (Hrsg.)

\section{Mehrsprachiges Lesetheater}

Handbuch zu Theorie und Praxis 
Mehrsprachiges Lesetheater 

Sabine Kutzelmann • Ute Massler • Klaus Peter • Kristina Götz • Angelika Ilg (Hrsg.)

\section{Mehrsprachiges Lesetheater}

Handbuch zu Theorie und Praxis

Verlag Barbara Budrich

Opladen • Berlin • Toronto 2017 
Bibliografische Information der Deutschen Nationalbibliothek

Die Deutsche Nationalbibliothek verzeichnet diese Publikation in der Deutschen Nationalbibliografie; detaillierte bibliografische Daten sind im Internet über http://dnb.d-nb.de abrufbar.

Gefördert durch

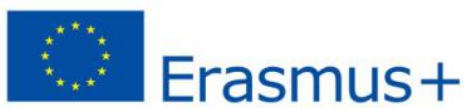

Schulbildung

Dieses Projekt wurde mit Unterstützung der Europäischen Kommission finanziert. Die

Verantwortung für den Inhalt dieser Veröffentlichung (Mitteilung) trägt allein der Verfasser; die Kommission haftet nicht für die weitere Verwendung der darin enthaltenen Angaben.

This project has been funded with support from the European Commission. This publication reflects the views only of the author, and the Commission cannot be held responsible for any use which may be made of the information contained therein.

Gedruckt auf säurefreiem und alterungsbeständigem Papier.

Alle Rechte vorbehalten.

C 2017 Verlag Barbara Budrich, Opladen, Berlin \& Toronto

www.budrich-verlag.de

$$
\text { ISBN 978-3-8474-2021-4 }
$$

eISBN 978-3-8474-1109-3

Das Werk einschließlich aller seiner Teile ist urheberrechtlich geschützt. Jede Verwertung außerhalb der engen Grenzen des Urheberrechtsgesetzes ist ohne Zustimmung des Verlages unzulässig und strafbar. Das gilt insbesondere für Vervielfältigungen, Übersetzungen, Mikroverfilmungen und die Einspeicherung und Verarbeitung in elektronischen Systemen.

Umschlaggestaltung: Bettina Lehfeldt, Kleinmachnow - www.lehfeldtgraphic.de

Lektorat und Satz: Ulrike Weingärtner, Gründau

Titelbildnachweis: Stillaufnahme aus dem MELT Film „LOUD, DEUTLICH, LENTEMENT: Mit

Mehrsprachigem Lesetheater Sprachgrenzen überwinden“ (s. Projektwebsite: http://melt-multilingualreaders-theatre.eu/ )

Druck: paper \& tinta, Warschau

Printed in Europe 


\section{Inhalt}

Das Projekt Mehrsprachiges Lesetheater: Ausgangslage, Zielsetzung

und praxisrelevante Ergebnisse der Entwicklung .................

Sabine Kutzelmann, Ute Massler und Robert Hendel

\section{Teil A}

\section{Forschungsmethodik}

1 Design-Based Research: Eine Forschungsstrategie zur Entwicklung nachhaltiger Innovationen in der schulischen Praxis . . . . . . . . . . . . Sabine Kutzelmann und Kristina Götz

2 Die schulischen Erprobungskontexte des MELT-Projekts . . . . . . . . . . . 32 Kristina Götz, Robert Hendel, Sabine Kutzelmann und Klaus Peter

3 Mehrsprachiges Lesetheater: Ergebnisse zur Akzeptanz dieser

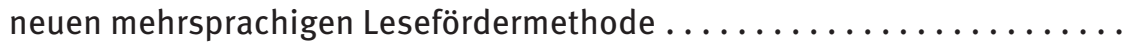
Kristina Götz, Sabine Kutzelmann und Ute Massler

Teil B

Theoretische Grundlagen des

Entwicklungs- und Forschungsprozesses

1 Leseflüssigkeit und Lesemotivation: Die beiden Förderdimensionen des Mehrsprachigen Lesetheaters . . . . . . . . . . . . . . . Kristina Götz und Sabine Kutzelmann

2 Das Unterrichtsdesign Mehrsprachiges Lesetheater aus linguistischer Sicht.

Klaus Peter und Dominik Unterthiner

3 Vom Readers' Theatre über interlinguale Literatur zum Mehrsprachigen Lesetheater: Die didaktisch-methodischen Leitlinien der Gestaltung im Kontext der Mehrsprachigkeitsdidaktik ................. Ute Massler und Sabine Kutzelmann

\section{Teil C}

\section{Das didaktisch-methodische Design:}

\section{Theoretische Grundlagen und Anleitung für die Praxis}

1 Mehrsprachige Lesetheaterstücke: Konzeption der Lehr-Lern-Materialien . . . Angelika Ilg, Franz Ludescher und Seraina Paul

2 Mehrsprachiges Vorlesen durch die Lehrperson als Design-Komponente des Mehrsprachigen Lesetheaters . . . . . . . . . . . . . . . . .

Ute Massler und Kerstin Theinert 
3 Miteinander sind alle Stars - Die Bedeutung des kooperativen Lernens

im Mehrsprachigen Lesetheater.

120

Sabine Kutzelmann und Kerstin Theinert

4 Literarisches Lernen als Design-Komponente des

Mehrsprachigen Lesetheaters . . . . . . . . . . . . . . . . .

Angelika Ilg und Kerstin Theinert

5 Die zentralen Lehr-Lern-Prozesse des Mehrsprachigen Lesetheaters:

Eine Anleitung für die Praxis

Sabine Kutzelmann, Ute Massler, Klaus Peter unter Mitarbeit von

Anne-Marie Antony, Stefanie Arnold, Kurt Benedikt, Andrea Büchel,

Stefanie Blodnig, Tessy Gollère, Robert Hendel und Christian Mertens

\section{Teil D}

\section{Curriculum Lehrerfortbildung}

1 Professionswissen und Einstellungen von Sprachlehrpersonen zur

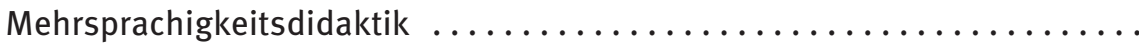
Klaus Peter und Dominik Unterthiner

2 Sprach- und Fachgrenzen überwinden: Die didaktische und strategische Konzeption des Fortbildungscurriculums zur Lesefördermethode

Mehrsprachiges Lesetheater ......................... 168

Sabine Kutzelmann und Ute Massler

Anhang 1) Anleitung zur Dramatisierung von mehrsprachigen Lesetheatern am Beispiel von Bend it like Beckham . . . . . . . . . . . . . . . . 181 Anhang 2) Lesetheater Heidi . . . . . . . . . . . . . . . . . . . . . . 185

Anhang 3) Lesetheater Luina - Die Seejungfrau vom Bodensee . . . . . . . . . . . 189

Anhang 4) Lesetheater Nasreddin Hodscha . . . . . . . . . . . . . . . . . . . . 191

Anhang 5) Lesetheater Dr Jekyll and Mr Hyde . . . . . . . . . . . . . . . . 195

Anhang 6) Toolbox: Dramapädagogik ...................... 196

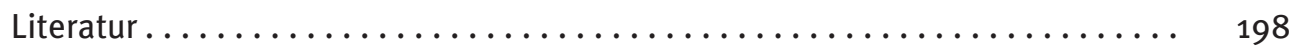

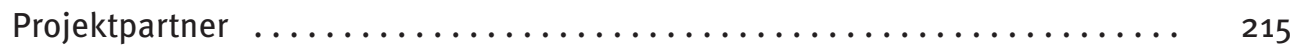

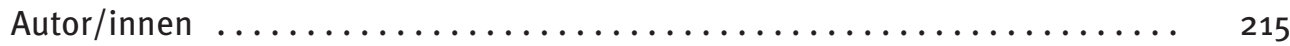




\title{
3 Mehrsprachiges Lesetheater: Ergebnisse zur Akzeptanz dieser neuen mehrsprachigen Lesefördermethode
}

\author{
Kristina Götz, Sabine Kutzelmann und Ute Massler
}

Ziel des Forschungsprojekts Mehrsprachiges Lesetheater (MELT) war es, ein innovatives, mehrsprachiges und praxistaugliches Unterrichtsdesign zur Förderung der Leseflüssigkeit und Lesemotivation für verschiedene Sprachenkombinationen zu entwickeln, das nachhaltig im Unterricht eingesetzt wird. In Kapitel A1, das die Untersuchung der Akzeptanz im Rahmen des Design-Based-Research-Ansatzes thematisiert, wurde die Bedeutsamkeit der Akzeptanz vonseiten der Lehrpersonen erläutert. So muss die Lehrperson das Unterrichtsdesign akzeptieren und es als nutzbringend und anwendbar ansehen, damit sie es im Unterricht wiederholt einsetzt. Nach Abschluss der Erprobungen wurden mit allen beteiligten Lehrpersonen Leitfadeninterviews durchgeführt und aus den Aussagen inhaltliche Kategorien nach Kuckartz (2012) gebildet. Aufgrund der kleinen Stichprobe (N=6 Lehrpersonen) wurde auf eine quantitative Auswertung innerhalb der Kategorien weitgehend verzichtet. Da MELT in sehr unterschiedlichen schulischen Kontexten erprobt wurde, wurden auch Beurteilungen einzelner Lehrpersonen als wichtig und nennenswert erachtet, da diese auf (kritische) Akzeptanz im jeweiligen, einzigartigen Kontext hinweisen. Im vorliegenden Kapitel wird zunächst, basierend auf dem beschriebenen Modell zur Handlungsvorhersage von Venkatesh et al. (2003; vgl. Teil A, Kap. 1), der erwartete Nutzen aus den Beurteilungen des Akzeptanzobjekts Mehrsprachiges Lesetheater hergeleitet (3.1). Dabei wird der erwartete Nutzen als das Ausmaß, zu dem die Verwendung der Lesefördermethode nach Ansicht der Lehrperson den Unterricht anreichert, definiert. Abschnitt 3.2 befasst sich mit dem erwarteten Aufwand der Nutzung von MELT, da er entscheidend die Nutzungsintentionen der Lehrpersonen beeinflusst. Außerdem wird auf die affektive Komponente eingegangen. Das emotionale Erleben, das mit einer Anwendung assoziiert wird, spielt im Modell von Venkatesh et al. zwar eine untergeordnete Rolle, fließt aber u. a. über den erwarteten Aufwand als Einfluss auf die Nutzungsintentionen ein. Soziale Einflüsse auf die Nutzungsintentionen der Lehrpersonen werden kurz in Abschnitt 3.3 besprochen, bevor sich Abschnitt 3.4 mit den Rahmenbedingungen befasst. Diesen kommt eine besondere Rolle zu, da sie im Modell von Venkatesh et al. die tatsächliche Nutzung einer Anwendung direkt und nicht über die Nutzungsintention beeinflussen. Die Nutzungsintentionen werden in Abschnitt 3.5 erläutert. Im abschließenden Fazit zum Mehrsprachigen Lesetheater als Lesefördermaßnahme in der Praxis (3.6) wird auf die geschilderten Ergebnisse unter Berücksichtigung der schulischen Erprobungskontexte (vgl. Teil A, Kap. 2) eingegangen. 


\subsection{Der erwartete Nutzen des Mehrsprachigen Lesetheaters}

Das Mehrsprachige Lesetheater als Gesamtkonzept wurde von allen Lehrpersonen sehr positiv beurteilt und als sehr praktikabel, strukturiert und umsetzbar beschrieben, wie folgende Aussage verdeutlicht: „Das Konzept finde ich sehr gut handhabbar, das passt, das ist stimmig (...) und praktikabel. Man muss es nur noch auf die Klasse, auf ihr Vorwissen abstimmen" (LP5, Z. 3-12). Lehrpersonen beschrieben es als ein Design, das den Lernenden Spaß macht und auch durch den Aspekt der Mehrsprachigkeit an ihre Lebenswelt anschließt. ${ }^{1}$ Zudem werden die Schüler/innen an Literatur herangeführt. Eine Lehrperson beschrieb den Mehrwert wie folgt:

Bei einem klassischen Lesetext oder einer Aufgabe muss nicht alles verstanden werden, um die Idee erschließen zu können oder eine Antwort zu geben. Mit MELT habe ich das Gefühl gehabt, dass es den Schüler/innen wichtig ist, auch Details zu kennen oder Gefühle interpretieren zu können, weil sie nachher ja die Rolle aufführen sollten. So wird für mich auf eine authentische Art und Weise ein tieferes Textverständnis gefördert. (LP 1, Z. 1164-168)

Nach Schwierigkeiten bei der Anwendung im Unterricht gefragt, nannten fünf der sechs Lehrpersonen u. a. ungünstige Rahmenbedingungen (vgl. 3.4). Zudem wurde bemerkt, dass das Design regelmäßig und fest im Unterrichtsjahr eingebaut werden sollte, um einen Mehrwert für das Lernen bei den Schüler/innen zu erzielen, wozu es ein hohes Maß an Engagement von den Lehrpersonen bedarf: „Damit MELT funktioniert, muss man sich darauf einlassen, und zwar ordentlich einlassen" (LP 3, Z. 10-11). Kritisch gesehen wurde von einer Lehrperson, dass durch den Fokus auf die Förderung der Leseflüssigkeit das Leseverständnis zurückblieb: „Wir haben gemerkt, dass die Schüler/innen nicht so viel verstehen, wie wir gedacht haben. Die lesen das einfach runter" (LP 6, Z. 158).

Die Lehr-Lern-Materialien: Insgesamt wurde die Auswahl der zur Verfügung stehenden mehrsprachigen Lesetheaterstücke von den Lehrpersonen als interessant, humorvoll und bezogen auf die Lernenden beschrieben. Geschätzt wurde besonders das breite Angebot der Texte mit unterschiedlichen sprachlichen Textniveaus und vielfältigen Themen, das eine Passung an die Klassen ermöglicht.

Zwei Lehrpersonen äußerten, dass ihre Klassen generell sehr gerne mit dem Unterrichtsdesign arbeiten bzw. für die Klasse das Rollenspiel im Vordergrund steht. Daher ist die Thematik der Texte vergleichsweise nebensächlich. Dessen ungeachtet wurde die Auswahl eines für die Klasse geeigneten Lesetheatertexts von allen Lehrpersonen als essenziell für das erfolgreiche Arbeiten dargestellt: „Der Lesetext ist das A und 0 von MELT" (LP 1, Z. 91).

Es kristallisierten sich vier Kriterien heraus, die bei der Auswahl der Texte beachtet werden sollten:

1.) Empfehlenswert ist, mit einfachen Texten zu starten und das Schwierigkeitsniveau langsam zu steigern.

1 Zitate der Lehrpersonen wurden im gesamten Handbuch zugunsten einer besseren Lesbarkeit sprachlich geglättet. Zudem wurde durchgehend die gender-neutrale Bezeichnung Schüler/innen eingesetzt. 
2.) Für schwächere Klassen sowie für den Einstieg in MELT sind Lesetheaterskripts mit in sich abgeschlossenen Szenen einfacher, da die Lernenden hier nicht zusätzlich noch den Zusammenhang zwischen den einzelnen Szenen verstehen müssen. Zudem sollten die Texte wenige und eindeutige Rollen mit klar umrissenen Charaktereigenschaften beinhalten.

3.) Um die motivierte Mitarbeit der Schüler/innen zu gewährleisten, sollten die Texte aus der Lebenswelt der Lernenden kommen, witzig und für sie gut verständlich sein.

4.) Die Lehrperson sollte mit dem (Buch-)Text vertraut sein und gerne damit arbeiten. Dies entlastet den Vorbereitungsaufwand und stärkt die Authentizität.

Die gleichzeitige Verwendung mehrerer Sprachen: Mehrsprachigkeit nimmt in den am Projekt beteiligten Ländern unterschiedliche Rollen ein (vgl. Teil A, Kap. 2). Für manche Lehrpersonen ist Mehrsprachigkeit Alltag oder wird in der Familie gelebt; andere sprechen hingegen meist nur eine Sprache. Daher wurde erwartet, dass die Lehrpersonen die gleichzeitige Verwendung mehrerer Sprachen im Unterrichtsdesign unterschiedlich akzeptieren. Vier Lehrpersonen waren gegenüber der Sprachenmischung offen, bei zwei anderen veränderte sich nach anfänglichen Bedenken die Einstellung zur gleichzeitigen Verwendung von mehreren Sprachen zum Positiven: „Also, ich finde es mittlerweile gut. Ich war am Anfang etwas skeptisch“ (LP 5, Z. 133). Zwei Lehrpersonen war es wichtig, dass eine Figur nur eine Sprache verwendet oder dass Figuren, die nahe verwandt sind (z. B. wenn sich Eltern mit ihrem Kind unterhalten), auch nur eine Sprache sprechen, um eine realistische Sprachsituation zu gewährleisten. Nach Ansicht der Lehrpersonen erleben die meisten Schüler/innen in ihrem Alltag Mehrsprachigkeit, vor allem in den gelebten Migrationssprachen: „Ich finde es sehr spannend, wie die Kinder damit umgehen. Die nehmen das sehr locker. (...). Das Miteinander der Sprachen ist kein Problem. Im Gegenteil, das passt sehr gut" (LP 6, Z. 149-154).

Es wurde als positiv empfunden, dass mehrere Sprachen gleichzeitig trainiert werden. Im Vergleich zum herkömmlichen Sprachenunterricht bietet das Mehrsprachige Lesetheater eine Lernumgebung, in der die Schüler/innen als Klasse gemeinsam aktiv und motiviert an der Fremdsprache arbeiten und besonders das laute Lesen trainieren: „Im Englischunterricht kommt das (Vor-)Lesen oft zu kurz, dass wirklich jede/r liest“ (LP 4, Z. 12-13). Zudem trainieren die Schüler/innen das Lesen in der Schulsprache, die in vier Kooperationsklassen für die Mehrheit der Schüler/innen nicht die Erstsprache ist.

Kritisch beurteilte eine Lehrperson, dass die Mehrsprachigkeit aber auch „einen Stolperstein" darstellt, insbesondere für die Aufführung vor einem Publikum, das nicht mit dem Text vertraut ist:

Und dann habe ich gemerkt, wenn wir mehrsprachige Lesetheater machen, können wir sie nicht vorführen. Vor den Eltern geht es noch, denn die wollen einfach ihre Kinder sehen oder hören. Aber den Inhalt verstehen? Da haben sie keine Chance. So konnten wir es auch nie einer Parallelklasse vorspielen. Die hätten die Geschichte nicht verstanden. Wenn man es immer wieder hört und die Geschichte kennt, dann geht es. Wir haben gemerkt, diese Mehrsprachigkeit ist eine Chance, aber auch ein Stolperstein. (LP 6, Z. 17-25)

Der Einbezug von Migrationssprachen: In einer Kooperationsklasse wurde bei jeder Erprobung mindestens eine der in der Klasse vorkommenden Migrationssprachen in das 
Mehrsprachige Lesetheater einbezogen, in zwei weiteren einmalig. Alle Lehrpersonen beurteilten dies sehr positiv, da sie wertschätzende Erfahrungen für die Schüler/innen, die die Migrationssprache sprechen, wahrnahmen. Besonders die Klassengemeinschaft profitiert nach Ansicht der Lehrpersonen davon, da die Schüler/innen dadurch offener für neue Sprachen und gegenüber ihren Mitschüler/innen werden:

Es war ein wertschätzender Moment und ein Anlass, mit den anderen Schüler/innen über die eigene Sprache zu sprechen. Eine bewusste Wahrnehmung der Schüler/innen mit Migrationshintergrund - aha, die können eine andere Sprache, was ja auch eine Leistung ist. Das ist den Schüler/innen nicht immer klar, dass da Leute in der Klasse sitzen, die Dinge können, über die man staunt. (LP 3, Z. 274-288)

Folgende Punkte, die beim Einbezug der Migrationssprache beachtet werden sollten, kristallisierten sich heraus:

- Nach Möglichkeit sollten die Schüler/innen mit Migrationssprache die entsprechenden Phrasen selbst übersetzen, um das Identifikationspotenzial und den Expertenstatus der Schüler/innen auszubauen.

- In den MELT-Texten sollten nur kurze und nicht zu viele Phrasen in einer Migrationssprache eingebaut werden, um die Verständlichkeit insbesondere für das Publikum bei der Aufführung nicht zu schmälern.

- Der Einbezug der Migrationssprache sollte zusätzlich zu und nicht auf Kosten der in der Schule geförderten (Fremd-)Sprachen erfolgen.

Der mehrschrittige Lehr-Lern-Prozess (vgl. Teil C, Kap. 5): Dieser wurde als stimmig, klar strukturiert, sinnvoll und übersichtlich bewertet, also insgesamt sehr positiv beurteilt. Mehrfach hervorgehoben wurde, dass der Ablauf aufgrund der wechselnden Sozialformen abwechslungsreich gestaltet ist. Zudem antizipieren die Schüler/innen die nächsten Arbeitsschritte nach mehrmaligen Durchführungen, was sowohl der Organisation als auch der Klassenführung zugutekommt.

Der Ablauf hat für mich etwas Ritualisierendes. Wie MELT eröffnet wird, wie die Erarbeitungsphase gestaltet wird, wie das nachher bei der Aufführung ist, mit Applaus und so. Das ist ritualisierend, und das gibt Sicherheit. Das ist das Schöne daran. Und ich habe gemerkt, dass sich die Schüler/innen nach einigen Durchführungen darauf freuen. (LP 1, Z. 345-348)

Unsere MELT-erfahrenen Lehrpersonen erklärten, dass der Lehr-Lern-Prozess besonders für unerfahrene Lehrpersonen „eine gute Unterstützung“ bietet. Hierzu passt auch, dass niemand einen entbehrlichen Prozessschritt nennen konnte. Positiv beurteilt wurde außerdem, dass der Lehr-Lern-Prozess „trotzdem noch Flexibilität lässt, um die eigene Identität und die eigenen Vorstellungen von Unterricht zu verwirklichen“ (LP 1, Z. 288-289). Um eine bessere Passung an Klasse, Lehrperson oder das jeweilige Lesetheaterstück zu erreichen, fassten Lehrpersonen manchmal einzelne Prozessschritte kürzer, gestalteten andere ausführlicher oder ließen auch einzelne Schritte verschmelzen. Zur Anpassung an die Lehrperson wurde geäußert:

Der Lehr-Lern-Prozess ist eine Grundvorlage, die Grundstruktur gibt. Das ist richtig und hilfreich, aber man wird nicht daran vorbeikommen, selber ein bisschen zu probieren, zu tüfteln, zu verändern und herauszufinden, was ist meines. (...). Es ist gut, dass es so 
eine Grundstruktur gibt, und ich würde allen wünschen, den Mut zu haben, das so zu probieren und zu machen, dass es nachher genau für sie passt. (LP 3, Z. 312-319)

Kritische Punkte in Bezug auf den Lehr-Lern-Prozess bezogen sich insgesamt eher auf die Rahmenbedingungen (z. B. Raumbelegung) und den Aufwand (in Anspruch genommene Zeit) als auf die grundlegende Konzeption (vgl. 3.2 und 3.4).

Mehrsprachiges Vorlesen durch die Lehrperson: Dies wurde als wichtiger und integraler Bestandteil des Lehr-Lern-Prozesses bezeichnet. Mehrfach wurde genannt, dass die Lehrperson hier als Vorbild bzw. als Modell für das gestaltende Vorlesen fungiert. Ferner bietet der Vorlesetext einen Einblick in das Lesetheater, der von der Lehrperson motivierend gestaltet werden kann und eine „Wohlfühlatmosphäre“ kreiert: „MELT steht und fällt mit diesem Einstieg. Je mehr die Schüler/innen hier begeistert werden können, desto leichter wird es fallen, MELT umzusetzen“ (LP 1, Z. 336-338).

Die mehrsprachigen Vorlesetexte sind - ebenso wie die Szenen für die Lernenden - in den unterschiedlichen Sprachkombinationen (Deutsch/Englisch/Französisch/2, Deutsch/Englisch, Deutsch/Französisch) gehalten. Für den Großteil der Lehrpersonen stellte dies keine besondere Herausforderung dar, da sie entweder die Fremdsprache in den Klassen unterrichten oder die verwendeten Sprachen in ihrem Alltag nutzen. So äußerte sich eine Lehrperson: „Ich konnte mich, so wie der Text gestaltet war, sehr gut damit identifizieren" (LP 1, Z. 365-366). In einer Kooperationsklasse wurde das mehrsprachige Vorlesen oftmals von der Deutschlehrkraft übernommen, die das mehrsprachige Vorlesen dennoch als sehr positiv bewertete:

Ich unterrichte Deutsch und habe mit Englisch nicht so viel zu tun. Trotzdem ist es gut, dass die Schüler/innen auch sehen: „Aha, die Person kann vielleicht auch nicht so gut Englisch, aber sie redet und traut sich." Da ist man ja auch Vorbild, dass man sagt, trotz Unsicherheiten machen wir das einfach so. (LP 3, Z. 138-143)

Gefragt nach ihrem emotionalen Befinden während des mehrsprachigen Vorlesens gaben drei der Lehrpersonen an, es anfangs komisch gefunden zu haben und Aufregung verspürt zu haben. Dies habe sich aber nach einigen Durchführungen gegeben, sodass das Vorlesen jetzt als normal oder sogar als Spaß oderGefallen bringend empfunden wird.

In Anbetracht dieser Beurteilungen ist es nicht verwunderlich, dass sich keine Lehrperson dafür aussprach, diese Komponente des mehrschrittigen Lehr-Lern-Prozesses zu streichen. Angemerkt sei hier, dass sich zwei der Lehrpersonen allerdings stark für kürzere Vorleseskripte einsetzten. Der Wert des Vorlesens durch die Lehrperson wurde wie folgt beschrieben:

Die Schüler/innen haben auch das Recht mal zuzuhören, von Mensch zu Mensch. Eltern machen das heute leider selten. Dieses Erleben, das muss man ihnen gönnen, und sie lernen sehr viel dabei. Da bin ich fast sicher, das ist überhaupt keine verlorene Zeit. (LP 6, Z. 516-521)

2 Es ist durchaus denkbar, auch in den Vorlesetext eine Migrationssprache zu integrieren, besonders wenn die Lehrperson diese Sprache spricht. 
Bedenken wurden geäußert, die das Hörverstehen der Schüler/innen betreffen: „Ich hatte manchmal ein bisschen die Angst, die Schüler/innen verstehen wichtige Stellen nicht" (LP 6, Z. 537-538). Allerdings ergaben die Unterrichtsbeobachtungen, die während der formativen Evaluation durchgeführt wurden (vgl. Teil A, Kap.1), dass die oben genannten Lehrpersonen diesen Bedenken konstruktiv begegneten. So wurden die Vorlesetexte in einer Klasse gekürzt und sprachlich an das Niveau der Klasse angepasst, um die Aufmerksamkeit der Schüler/innen beim Vorlesen zu halten. In einer anderen Klasse wurde die sprachliche und inhaltliche Einführung in das Lesetheater intensiviert, um das Niveau der Klasse anzusprechen.

Kooperatives Lernen: Alle Lehrpersonen erachteten das kooperative Lernen als wichtige, anspruchsvolle Lernform. Lehrpersonen und Klassen, die über Erfahrungen mit dieser Lernform verfügten, fiel das selbstständige Arbeiten in Gruppen leicht: „Wir arbeiten schon länger mit kooperativen Lautleseverfahren, die Schüler/innen waren diese Arbeitsstrukturen gewohnt. Das hat sehr gut funktioniert" (LP 3, Z. 381-383).

Es kristallisierte sich jedoch heraus, dass bei unerfahrenen Gruppen eine sorgsame, zeitintensive Einführung und Einarbeitung nötig ist. Dann können sie aber davon in anderen Lernkontexten profitieren:

Also, anfangs war es ganz, ganz schwer. Die Schüler/innen haben sich nicht wirklich gegenseitig geholfen am Anfang. Das war wirklich ein großer Schritt denen beizubringen, okay, ihr seid jetzt eine Gruppe, ihr müsst euch gegenseitig helfen. (...). Das wurde schon bei der ersten Durchführung besser. Man muss den Schüler/innen wirklich mal zeigen, wie man sich gegenseitig Feedback gibt, dass man sich zuhören muss und nicht einfach nur darauf wartet, dass seine Zeile jetzt kommt. Man muss aufmerksam zuhören, wie die anderen es machen, und eben denen helfen, wie sie es anders machen können. Im Nachhinein finde ich nach all den Durchführungen, dass sie viel, viel besser geworden sind, auch jetzt bei anderen Sachen. (LP 2, Z. 385-394)

Des Weiteren wurde die Arbeitshaltung mancher Lernender kritisiert: „Wenn man nicht im Raum war, haben gewisse Kinder nicht effektiv und gut gearbeitet" (LP 6, Z. 585586). Abhilfe dafür versprechen sich die Lehrkräfte vor allem von der Unterstützung durch eine zweite Lehrperson und durch eine bessere räumliche Situation während der kooperativen Phasen (vgl. 3.4). Zudem hängt das erfolgreiche Arbeiten in Gruppen von den Schüler/innen und deren kooperativen Fertigkeiten ab: „Es gibt Kinder, die können gar nicht in Gruppen arbeiten, andere sind sehr gute Leader" (LP 6, Z. 550-551). Zum anderen hängt das Arbeiten aber auch von dem jeweiligen Prozessschritt, in dem Gruppenarbeit verlangt wird, ab. So differenzierte eine Lehrperson:

Ich habe die Gruppen besonders in dieser Anfangsphase sehr aktiv erlebt: wenn es darum geht, Rollen zu finden und zu beschließen, wer was tut. In der Erarbeitungsphase, also wenn es darum geht, die Texte wirklich im Detail durchzulesen, richtig zu artikulieren, also wirklich die Leseflüssigkeit zu steigern, das war teils etwas schwieriger. Wenn ich die Schüler/innen aufgefordert habe, den Text zum vierten, fünften Mal zu lesen, das ist schon dann etwas ins Stocken geraten und ich habe dann auch das Gefühl gehabt, dass ich zwischen den Gruppen so ein bisschen hin und her gelaufen bin, um dann immer wieder anzustubsen und zu stimulieren. (LP 1, Z. 404-410)

Literarisches Lernen: In drei Kooperationsklassen wurden Formen des literarischen Lernens (Anschlusskommunikation und dramapädagogische Übungen) erprobt, die 
die Schüler/innen anregen sollen, über die Geschichte und die Figuren zu sprechen, sich in diese handlungsorientiert einzufühlen und Zusammenhänge zwischen dem Gelesenen und ihren eigenen Erfahrungen herzustellen. Die Lehrpersonen bewerteten diese Gespräche als wichtig, da die Schüler/innen im Unterricht sonst kaum Gelegenheit haben, sich über den Lesestoff auszutauschen: „Je öfter die Schüler/innen die Gespräche geführt haben, umso mehr Sinn hat es gemacht. Je länger sie dabei sind, desto mehr konnten sie auf den Inhalt achten und umso mehr waren sie kritikfähiger" (LP 5, Z. 623-625). Zusätzlich bringen die Gespräche Abwechslung, „sodass MELT nicht jedes Mal gleich verläuft“ (LP 6, Z. 646-647). Allerdings „passt das literarische Gespräch nicht bei allen Geschichten gleich gut (LP 6, Z. 685).

Deutlich wurde in den Gesprächen, dass das literarische Lernen zwar eine wichtige, aber auch herausfordernde Komponente des Mehrsprachigen Lesetheaters darstellt. Zum einen wurde berichtet, dass es schwierig ist, dafür den geeigneten Zeitpunkt zu finden. Eine weitere Lehrperson betrachtete es als Überfrachtung des Designs, indem zwei dezidierte Lernziele, die Förderung der Leseflüssigkeit und des literarisches Lernens, darin vereint werden, und tendierte daher dazu, nur jeweils einen Schwerpunkt zu setzen:

Die Aufführung gut hinzubekommen, dass die Kinder gut sprechen, gut vortragen: Das ist ein riesiges Ziel. Und der Zugang zur Literatur und die Kommunikation darüber, was jetzt passiert ist, das ist eigentlich auch wieder ein riesiges Ziel. Und da setzt man zwei riesige Ziele in ein Skript hinein. Und ich merke, wenn ich beides erfülle, dann kommt entweder das eine zu kurz, oder das andere. So bin ich fast geneigt zu sagen, jetzt geht es um das Lesetheater, und nächstes Mal ist das Lesetheater Anlass, um das literarische Lernen breiter anzulegen und vertieft durchzuführen. (LP 3, Z. 476-485)

Für diese Lehrperson ist eine alternative Herangehensweise, das literarische Gespräch quasi durch die dramapädagogischen Übungen stattfinden zu lassen:

Die Schüler/innen tauchen mithilfe der dramapädagogischen Übungen wirklich in Figuren ein, übernehmen ihre Rolle, argumentieren, wie diese Rolle argumentieren würde und das funktioniert (...) und macht den Schüler/innen Spaß. (...). Da muss man dann nicht im Nachhinein künstlich über irgendwelche Inhalte von Geschichten reden oder über irgendwelche Aussagen von Geschichten sprechen. Das kriegt man mit, wenn diese Standbilder reden oder beim heißen Stuhl jemand erzählt, wer er ist und was ihn da bewegt hat, seine Handlungen dann erklärt, dann taucht man in die Geschichte eigentlich ein. (LP 3, Z. 441-453)

Die dramapädagogischen Übungen wurden durchgehend als sinnvoll und wichtig für die inhaltliche Erarbeitung der Lesetheaterstücke, die Motivation der Lernenden und das Üben des deutlichen Sprechens beurteilt. Auch hier wurde von allen Lehrpersonen die Passung von Lesetheaterstück, Klasse und Übung besprochen. Ein Kriterium für die Passung ist das Alter der Schüler/innen:

Es kommt immer auf das jeweilige Stück an. Einige Übungen eignen sich für jüngere Schüler/innen gut, weil sie das einfach noch machen. Die Achtklässler waren nicht mehr so bereit, guckten eher, was die anderen machen. Da behindert das vielleicht sogar eher. (LP 5, Z. 587-591) 
Ähnlich wie beim kooperativen Lernen handelt es sich um eine Lernform, die in der Klasse frühzeitig etabliert werden sollte, denn „wenn man bzw. die Schüler/innen solche Arbeitsweisen nicht gewohnt sind von Anfang an, dann wird das schwierig. Das müssen die ein bisschen kennen, gewohnt sein, und dann ist das unglaublich fruchtbar" (LP 3, Z. 446-447).

Wirkung auf die Klasse: Ohne Ausnahme berichteten die Lehrpersonen, dass das Design bei ihren Klassen gut angekommen ist. Die Schüler/innen freuten sich auf den Unterricht, waren motiviert, arbeiteten gut mit und fanden die Geschichten meist spannend. Zwei Lehrpersonen berichteten, dass die Motivation und die Mitarbeit immer besser wurden (in einem Fall, nachdem die Klasse zunächst als skeptisch gegenüber dem „neuen“ Unterricht erlebt wurde), je mehr die Abläufe bekannt waren und verinnerlicht wurden. Eine andere Lehrperson sah die Attraktivität der Methode darin, dass sie sich vom herkömmlichen Unterricht unterscheidet:

Die Schüler/innen haben selber am Anfang gesagt, das ist kein normaler Unterricht. Sie müssen nichts schreiben, sie müssen lesen und anstreichen, und dann ist es für die immer so ein bisschen Freiheit, wenn sie im Haus rumlaufen dürfen und da selber etwas machen. (LP 5, Z. 717-721)

Als negativ bewerteten zwei Lehrpersonen, dass manchen Schüler/innen die Arbeit zu laut gewesen ist:

Es gibt einige Schüler/innen die sagen, ihnen ist es zu laut im Raum. (...). MELT bietet viele Ablenkungsmöglichkeiten: Es gibt viele Reize, viele Reaktionen, vieles, das im Raum passiert, und natürlich fällt es manchen Schüler/innen entsprechend schwerer, sich zu fokussieren. (LP 1, Z. 640-645)

Diese Beobachtungen beziehen sich auf Probleme, die durch die Rahmenbedingungen (vgl. 3.4) entstehen. Es ist möglich, dass förderlichere Rahmenbedingungen wie z. B. mehrere, zur Verfügung stehende Räume und weitere Lehrpersonen zur besseren Betreuung der Gruppen diese Probleme mildern bzw. beseitigen.

Für die Einschätzung des Nutzens ist wichtig, inwieweit die Lehrperson im Unterrichtsdesign potenzielle Lehr- und Lernziele erkennt und diese als erreichbar einstuft. Diesbezüglich äußerte sich die Mehrheit der Lehrpersonen eher verhalten; die Leseflüssigkeit habe sich im Laufe der Erprobungen eher nicht merkbar verändert. Wahrgenommene Verbesserungen wurden eher dem generellen Entwicklungsverlauf als der Lesefördermethode zugeschrieben. Die meisten Lehrpersonen schränkten jedoch ein, dass sie die Methode nicht oft und lange genug angewendet hatten, um eine eindeutig wahrnehmbare Verbesserung der Leseflüssigkeit zu erzielen. Vereinzelt wurde in der Fremdsprache bzw. bei schwächeren Schüler/innen eine verbesserte Leseflüssigkeit bemerkt.

In Bezug auf die Lesemotivation gaben die meisten Lehrpersonen an, dass ihre Schüler/innen generell eher wenig lesen und sich das nicht sichtbar verändert hat. Eine andere Lehrperson bemerkte vielmehr eine Veränderung in der Einstellung gegenüber Texten:

Ich merke einen Unterschied in ihrer Bereitschaft, sich mit Texten auseinanderzusetzen. Sie sind motivierter, sich mit geschriebener Sprache auseinanderzusetzen. 
Sobald sie in einer kleinen Gruppe an einem Text arbeiten können, ist eine positive Dynamik zu spüren, die, denke ich, sehr entscheidend von MELT beeinflusst wurde. (LP 1, Z. 602-606)

Betrachtet im Licht von empirischen Befunden zur Förderung von Leseflüssigkeit und Lesemotivation (vgl. Teil B, Kap. 1) sehen wir hier unseres Erachtens dennoch ein ermutigendes, da realistisches Bild: In Anbetracht der kurzen Erprobungen, die zudem mit beachtlichem Abstand zueinander stattfanden, waren in der Tat keine großen Veränderungen bei Lesemotivation und Leseflüssigkeit zu erwarten. Es entstand jedoch ein klares Bild bezüglich der generellen Akzeptanz, das bei Schüler/innen und Lehrpersonen durchweg positiv ausfiel.

Zwei weitere Lernziele wurden häufig genannt: Mehrheitlich meinen die Lehrpersonen, dass das soziale Lernen insbesondere durch die Arbeit in Gruppen gefördert wird (vgl. Abschnitt 3.5). Zudem schätzen alle Lehrpersonen sehr, dass das Präsentieren als mündliche (Kern-)Kompetenz intuitiv mit MELT geschult wird:

Dem Schulleiter ist aufgefallen, dass unsere Klasse extrem gut auftreten kann. Vor vielen Leuten laut reden, in Schülerversammlungen und so. Sie haben keine Hemmungen, und ich finde das so wichtig. Heute musst du dich verkaufen können. Und das können sie jetzt wirklich gut. (LP 6, Z. 812-817)

Die hier ausgeführte Diskussion des Akzeptanzobjekts Mehrsprachiges Lesetheater wird in Bezug auf den erwarteten Nutzen ausführlich im Fazit (3.6) besprochen.

\subsection{Der erwartete Aufwand für das Mehrsprachige Lesetheater}

Erwarteter Aufwand: Der Aufwand in Bezug auf die Vorbereitung wurde weitgehend als angemessen bewertet: „Mit diesem ,Rezeptbuch' (methodisch-didaktischer Kommentar), in dem alles kurz und genau erklärt ist, ist MELT machbar" (LP 4, Z. 643-644). Eine weitere Lehrperson 5 lobte, dass die Vorbereitung aufgrund der schon vorhandenen Materialien nicht aufwändig ist:

Ich muss mir halt Überlegungen zu den Sozialformen und den Einstig machen, so wie ich es immer mache. Das Material ist ja vorhanden. Ich muss mir im Prinzip nur noch selber genau überlegen, wie ich das machen möchte, und von dem her ist das ganz angemessen so. (LP 5, Z. 798-803)

Eine Lehrperson hob positiv hervor, dass sich vieles für sie intuitiv und ohne besondere Vorbereitung ergibt: „Viele gute Ideen kommen ja auch erst, wenn ich die Kinder sehe. Da muss ich mir nicht lange voraus überlegen, wie wir die Szene gut spielen könnten“ (LP 6, Z. 1019-1021). Ebenso angetan zeigte sich eine Lehrperson von der Anleitung, wie die Lesetheater im Unterricht eingesetzt werden können:

Es gibt dieses, ich sage jetzt „Rezeptbuch“, in dem die ganzen Methoden vorgestellt sind, dann ist es nicht so schwer für mich, gerade als junge Lehrerin. Dann ist es sicher hilfreich und nicht zu aufwändig, wenn das kurz und knapp erklärt wird. (LP 4, Z. 627-634)

Der Aufwand für die Durchführung wird hingegen höher eingeschätzt, dieser kann aber teils auch durch die Lehrperson selbst bestimmt werden: 
Es liegt in der Freiheit der Lehrperson, die Einführung, das Vorlesen aufwändiger zu gestalten. (...). Deswegen wäre das jetzt nicht mehr oder weniger Aufwand, als wenn ich einen anderen Unterricht vorbereite. Die Durchführung ist aufgrund der Faktoren, Zeit, Personal, räumliche Ressourcen aufwändiger. In der Nachbereitung finde ich es auch nicht aufwändig. Es hat Potenzial, damit ich es mit anderen Unterrichtsinhalten verknüpfen kann. (LP1, Z. 698-705)

Eine andere Lehrperson jedoch bewertete das Design als aufwändig und begründete diese Einschätzung in erster Linie mit dem Aufwand, die Maßnahme neu in einer Klasse einzuführen. Um dies erfolgreich zu tun, müssen zunächst die Arbeitsformen (z. B. Gruppenarbeit) gut in der Klasse eingeführt werden: „Bevor das nicht klar ist, dass das funktioniert und gut funktioniert, finde ich es ganz schwierig, mit MELT zu arbeiten“ (LP 3, Z. 703-704).

Der zeitliche Aufwand für die Durchführung wurde von einigen Lehrpersonen angesprochen:

Es ist halt zeitintensiv. Ich weiß nicht, inwiefern das umsetzbar ist für alle Klassen, dass man sich da wirklich sechs Stunden oder wie auch immer nimmt, um das umzusetzen. (LP 2, Z. 649-651)

Eine Lehrperson fasste ihre Gedanken zum Aufwand folgendermaßen zusammen: „Der Aufwand ist es wert, wenn man schaut, was dabei rauskommt" (LP 2, Z. 648).

Emotionales Erleben: Die Erprobungen fanden unter durchgehender Beobachtung der Forschenden statt, was, zusammen mit dem dahinterstehenden organisatorischen Aufwand, von den Lehrpersonen als eher stressig empfunden wurde. Wenn die Lehrpersonen jedoch dezidiert gefragt wurden, wie sie sich mit MELT, ungeachtet der durch die Erforschung bedingten Rahmenbedingung, fühlten, so beschrieben sie durchgehend positive Emotionen: „Jetzt denke ich, ich kann sehr gut damit arbeiten, ich fühle mich wohl dabei“ (LP 1, Z. 549-550). Hier fließt eine zeitliche Komponente mit in die Bewertung ein. Die Aussagen mehrerer Lehrpersonen deuten an, dass sich ihr emotionales Erleben im Laufe der Erprobungen zum Positiven entwickelt hat:

Für mich ist das Arbeiten mit MELT eigentlich nur positiv. Ich weiß inzwischen, was ich von MELT will, ich weiß, was ich von den Kindern will, und ich weiß auch, damit umzugehen. (LP 3, Z. 537-539)

Anfangs war ich sehr aufgeregt (...). Ich wusste nicht, was auf mich zukommt. Ich kannte das Ganze noch nicht, ich wusste nicht, wie die Kinder darauf reagieren, ob die das überhaupt umsetzen können und wollen, ob sie motiviert sind. Ich war am Anfang eher skeptisch, weil ich auch nicht wusste, wie unsere schwachen Schüler/innen mit den Sprachen klarkommen. Aber es wurde von Mal zu Mal wirklich besser, und es hat Spaß gemacht. Ich sehe auch wirklich, dass es etwas gebracht hat. Das ist das Wichtigste. (...). Ich habe ein ganz positives Gefühl gegenüber MELT. (LP 2, Z. 493-510)

Für eine weitere Person war die Wirkung, die die Anwendung auf ihre Klasse hat, eine Determinante ihres eigenen Wohlbefindens: „Ich habe mich eigentlich schon wohl damit gefühlt, vor allem, weil die Schüler/innen es auch gerne gemacht haben“ (LP 5, Z. 639-641). Schließlich stärkte das Arbeiten im Tandem mit einer lesetheatererfahrenen Lehrperson das positive Empfinden einer Lehrperson: „Ich hatte immer Sicherheit, 
weil die andere Lehrperson dabei war. Das war für mich schon wichtig. (...). So war es für mich sehr angenehm, da hineinzurutschen“ (LP 4, Z. 497-502).

\subsection{Soziale Einflussfaktoren}

Hier ergab sich ein einheitliches Meinungsbild. Alle Lehrpersonen gaben an, dass sie es erwarten, von der Schulleitung unterstützt zu werden, und bisher es so auch erfahren haben. Eine Lehrperson konnte sich tatkräftige Unterstützung seitens der Schulleitung vorstellen, z. B. könnte diese eine zweite Lehrperson zu Verfügung stellen, die Krankheitsvertretungsstunden übernimmt, aber nicht anderweitig gebraucht wird: „Organisatorisch wäre da auch eine Stundenplanänderung oder ein Tausch mit Kolleg/innen kein Problem" (LP 5, Z. 909-915).

Alle Lehrpersonen hatten bereits mit Kolleg/innen über das Mehrsprachige Lesetheater gesprochen. In den Kollegien war ein Interesse an Weiterbildungen da (im Falle eines Partners wurde es von einigen Kolleg/innen bereits wahrgenommen). Eine Lehrperson vermutete, dass mit den neuen Lehrmitteln das Interesse vieler Kolleg/innen geweckt wird. In zwei Fällen wurde berichtet, dass Kolleg/innen bereits im Unterricht mit dem Mehrsprachigen Lesetheater hospitiert haben, in einem weiteren Fall wurde es angeboten:

Ich würde es auf jeden Fall weiterempfehlen und auf jeden Fall sagen, mach zwei bis drei Versuche, bevor du eine Entscheidung triffst. Das ist ja immer so, wenn was Neues gemacht wird, am Anfang ist es immer mehr Aufwand und auch eine etwas unwohle Situation. (...) Bei Rückfragen stehe ich gerne zur Verfügung oder wäre auch gerne bereit, vielleicht einen Durchlauf mitzumachen, dass wir die Klassen mischen, oder du kommst mal zu mir, ich komm mal zu dir, im Sinne einer kollegialen Hospitation. Das würde ich sehr sinnvoll empfinden. (LP 1, Z. 812-821)

So berichteten alle Lehrpersonen, dass die Anwendung des Mehrsprachigen Lesetheaters in deren Arbeitsumfeld mit Interesse und Unterstützung der Kolleg/innen begleitet wurde, was auf einen positiven sozialen Einfluss schließen lässt.

\subsection{Rahmenbedingungen}

Es kristallisierten sich drei „ideale“ Rahmenbedingungen heraus, unter denen die Lesefördermaßnahme Mehrsprachiges Lesetheater gut anwendbar ist: (1) ausreichend Räumlichkeiten, (2) Unterstützung durch eine zweite Lehrperson (3) und ausreichend Zeit.

Alle Lehrpersonen äußerten, dass genügend Räume zur Verfügung stehen müssen, damit die einzelnen Gruppen relativ ungestört arbeiten können. Ansonsten führt das Arbeiten in nur einem Klassenzimmer zu Lautstärkepegeln, unter denen die Schüler/innen nicht produktiv arbeiten können: „Das Arbeiten in einem Raum geht auch. Aber es ist nicht ideal, und es ist auch belastend für einen selber und für die Schüler/innen, wenn es laut wird, und die müssen konzentriert etwas üben“ (LP 5, Z. 639641). 
Dies bedingt die nächste Rahmenbedingung: ausreichend Personal bzw. im besten Fall eine zweite Lehrperson, die den Unterricht mitbetreut. So kann einerseits der Aufsichtspflicht nachgekommen und für Disziplin gesorgt werden, andererseits können die selbstständig arbeitenden Gruppen besser betreut und angeleitet werden.

Das Problem war, dass man nicht immer individuell helfen konnte. Wir haben so viele schwache Schüler/innen, dass die stärkeren nicht ausreichen, um die schwachen mitzunehmen. So musste ich mich immer in hundert Teile teilen, damit ich jedem gerecht werden konnte. (LP 2, Z. 23-25)

Allerdings merkten zwei Lehrpersonen an, dass das Unterrichten im Tandem wiederum (zeit)aufwändige Absprachen benötigte: „Ich habe sehr viel Unterricht und keine Freistunden, da war es sehr schwierig, Stunden zusammenzulegen. Deshalb haben wir MELT ein bisschen aufgeteilt" (LP 4, 45-48). Der Faktor Zeit wurde von den erfahrenen Lehrpersonen eingehend diskutiert. Drei Lehrpersonen betonten, dass es die Möglichkeit geben sollte, mit der Klasse am Stück am Mehrsprachigen Lesetheater zu arbeiten. Für eine Lehrperson war es wichtig, die Fördermethode intensiv über zwei Wochen hinweg durchzuführen, um damit intensiver an den Texten, am Leseverständnis und an der Sprachgestaltung arbeiten zu können.

Wenn ich mir zwei Wochen Zeit nehme, geht es vielleicht mehr in die Tiefe, und die Kinder könnten noch mehr profitieren. (...). Ich habe manchmal das Gefühl, es passiert auch viel, wenn man nicht unmittelbar am Üben ist. Der Prozess dazwischen ist auch wichtig. Und dazu gibt es sonst keine Möglichkeit. Es ist immer Schlag auf Schlag, gerade wieder der nächste Schritt. Und wenn man ein bisschen Zwischenzeit gäbe, nur schon einen Tag, ob dann nicht auch was passieren würde? (LP 6, 416-418, 454-464)

Nach dem Akzeptanzmodell fließt neben den oben genannten Faktoren auch die Kompatibilität der Anwendung in diese Variable ein. Bemerkenswert ist, dass alle Lehrpersonen die Lesefördermethode als kompatibel mit ihrem jeweiligen Lehrplan sahen.

MELT gibt mir eigentlich eine sehr sinnvolle und authentische Methode, wie ich das aktive Nutzen der Sprache, das Hörverstehen und das Leseverstehen fördern kann. Deswegen ist MELT super kompatibel mit dem Lehrplan. Es ist eine tolle Methode, um vom schriftlichen Gebrauch der Sprache ein bisschen abzuweichen und ein bisschen vielfältiger zu unterrichten. (LP 1, Z. 760-764)

Auch sahen die Lehrpersonen, dass die Methode an den sonstigen Unterricht anschlussfähig ist, der durch die eingeführten sozialen Arbeitsformen bereichert werden kann. Außerdem würden laut einer Lehrperson die Schüler/innen im Unterricht von den erworbenen Kompetenzen im Präsentieren profitieren: „Dieses Selbstwertgefühl, dieses gute Gefühl, ich kann etwas laut erklären und sagen, das nutzt ja überall“ (LP 6, Z. 1117-1119).

\subsection{Nutzungsintentionen}

Erfreulicherweise teilten vier der Lehrpersonen mit, dass sie planen, Mehrsprachiges Lesetheater im Laufe des neuen Schuljahres mit ihren Klassen zu verwenden: 
Es wurde uns freigestellt, ob wir es (MELT) einsetzen wollen oder nicht, und wir wollen das unbedingt machen. Wir werden vielleicht unsere Projektstunden dafür nehmen. Das heißt, das wären dann zwischen zwei und vier Stunden pro Woche und das verteilt auf ein ganzes Trimester oder auf sechs Wochen. (LP 2, Z. 726-730)

Zwei Lehrpersonen wollen das Unterrichtsdesign in der 5. Klassenstufe einsetzen:

Ich denke, dass es gerade in Klasse 5 gut einsetzbar ist. Ich arbeite mit drei Kolleg/ innen sehr eng zusammen. (...). Wenn alle sagen, wir würden das mal machen, könnten alle gemeinsam das vorbereiten, wissen, wie es abläuft und auch vielleicht gekoppelt daran arbeiten. (LP 5, Z. 924-929)

Eine weitere Lehrperson möchte die Lesefördermethode gezielt mit einer Gruppe eher lernschwacher Schüler/innen einsetzen:

Ich habe beispielsweise in Französisch eine Gruppe, die ist eigentlich sehr, sehr lernschwach in Französisch, und ich denke, dass es bei den Schüler/innen wenig Sinn macht, sich die Sprache ständig über den schriftlichen Gebrauch anzueignen. Und MELT eignet sich da hervorragend, um sich spielerisch der Sprache zu nähern. (...). Wenn wir da zweisprachig arbeiten, damit sich die Schüler/innen schneller den Kontext erschließen können, die Sprache verstehen, ist das eine super Methode. Und das werde ich auf jeden Fall versuchen. (LP 1, Z. 777-779)

Die zwei verbleibenden Lehrpersonen nannten pragmatische Gründe dafür, warum sie die Methode im neuen Schuljahr nicht anwenden können, z. B. weil nicht genug Schulstunden zur Verfügung gestellt wurden, oder nur Stunden in der Abschlussklasse gehalten wurden, in der eine Einführung des Designs nicht sinnvoll und zeitlich nicht möglich ist:

Ich habe im Moment leider keine Klasse, mit der ich das machen kann. In der letzten Klasse einführen finde ich persönlich nicht sinnvoll. Bis wir die Methoden eingeübt haben, ist das Schuljahr vorbei. Vor allem ist in der 4. Klasse so viel anderes zu machen. In der 1. Volksschulklasse ist es zu früh. (...). Sollte ich das nächste Jahr wieder in die jetzige Zweite einsteigen dürfen, kann ich mir das schon vorstellen einmal auszuprobieren, wie MELT mit denen funktioniert. (LP 4, Z. 721-733)

Beide Lehrpersonen gaben aber an, interessierte Kolleg/innen in das neue Unterrichtsdesign einführen zu wollen, und tragen dadurch dazu bei, dass es in der Praxis tatsächlich genutzt wird.

\subsection{Fazit}

Lehrpersonen sehen einen deutlichen Nutzen in der Anwendung des Mehrsprachigen Lesetheaters. Die Lehrpersonen schätzen das klar strukturierte, abwechslungsreiche didaktisch-methodische Design mit seinen leicht verfügbaren, dramatisierten Texten, die viele unterschiedliche Themen und Textniveaus bieten. Die Mehrsprachigkeit erscheint überwiegend unproblematisch und bietet nach Ansicht der Lehrpersonen im Vergleich zum herkömmlichen Sprachenunterricht einen deutlichen Mehrwert (,relative advantage“, Venkatesh et al. 2003: 449) für das Lesetraining in Schul- und Fremdsprachen. Die Integration von Migrationssprachen wird sehr positiv beurteilt, da dadurch die 
Schüler/innen mit Migrationshintergrund Wertschätzung erfahren und die Klassengemeinschaft davon durch eine größere Offenheit gegenüber den Klassenkamerad/innen und Sprachen allgemein profitiert. Das Vorlesen durch die Lehrperson wird in seiner Funktion als Vorlesemodell und als motivierende Hinführung auf die Arbeit mit den Lesetheaterskripts als eine der Kernkomponenten des Unterrichtdesigns geschätzt. Dem kooperativen und literarischen Lernen stehen die Lehrpersonen wertschätzend gegenüber, sind sich der Herausforderungen dieser Lernformen jedoch bewusst. Einzelne Aspekte des kooperativen Lernens, z. B. das Feedbackgeben und kooperierende Lautleseverfahren, sollten daher vorgeschaltet oder parallel eingeführt werden, wenn die Klasse oder die Lehrperson damit noch keine Erfahrungen hat. Das literarische Gespräch erweitert den zeitlichen Rahmen und kann abhängig vom jeweiligen Lesetheaterstück aufgegriffen und ausgebaut werden. Die Wirkung des Mehrsprachigen Lesetheaters auf die Klasse wird weitgehend positiv beurteilt, und bei regelmäßiger und häufiger Anwendung halten die Lehrpersonen die Förderung der Leseflüssigkeit für möglich. Des Weiteren wurden wichtige Kernkompetenzen, wie das „Sich-Präsentieren“ und „soziales Lernen" genannt, die mit der Anwendung des Designs gefördert werden.

Die Lehrpersonen fühlen sich bei der Anwendung von MELT gut. Dieses Wohlbefinden wird dadurch verstärkt, dass sie eine positive Wirkung der Lesefördermethode auf die Klasse wahrnehmen.

Während das Design in Bezug auf die Vor- und Nachbereitung als nicht aufwändig beurteilt wird, stellt der Aufwand, der mit der Durchführung (und mit der Einführung von Arbeitsformen in der Klasse) assoziiert wird, eine Herausforderung dar. Besonders der zeitliche Aspekt wird auch in anderen Zusammenhängen oft als „,kritischer" Punkt genannt (vgl. 3.1; 3.4). Nach Ansicht der Lehrpersonen können bestimmte Rahmenbedingungen den Aufwand jedoch mildern. Die Kompatibilität mit den Lehrplänen und die Anschlussfähigkeit zum weiteren Unterricht sind gegeben und begünstigen die Anwendung im Unterricht.

Der Einsatz des Mehrsprachigen Lesetheaters wird von Interesse und Unterstützung der Kolleg/innen begleitet, was auf einen positiven sozialen Einfluss schließen lässt. Sehr erfreulich ist des Weiteren, dass die Lehrpersonen deutliche Nutzungsintentionen zeigen und beabsichtigen, MELT weiter zu verwenden.

Abschließend soll noch eine Einschränkung der Akzeptanzüberprüfung thematisiert werden: Im Sinne des engen Verständnisses der Akzeptanzforschung können keine Aussagen über die längerfristige Nutzung der Maßnahme gemacht werden. Da die summative Evaluation unmittelbar nach Abschluss der schulischen Durchführungsphase stattgefunden hat, kann lediglich die Nutzungsintention überprüft werden, aber nicht das tatsächliche zukünftige Nutzungsverhalten. Es können deshalb keine Aussagen darüber getroffen werden, ob die Lehrpersonen auch nach Beendigung des Projekts das Mehrsprachige Lesetheater mit ihren Klassen wieder anwenden werden. In der Forschungsliteratur wird jedoch eine hohe Korrelation zwischen den Nutzungsintentionen und dem tatsächlichen Nutzungsverhalten angenommen (siehe Fishbein/Ajzen 2010: 43-72 für eine Diskussion zur Vorhersage von Verhalten), sodass davon auszugehen ist, dass die Nutzungsintentionen der Lehrpersonen weitgehend zu einer Nutzung des Designs im Unterricht führen werden.

Zwei methodologische Punkte seien noch zur Akzeptanzstudie genannt, die die Sicht der Lehrpersonen auf das Unterrichtsdesign beeinflusst haben könnten. Die For- 
schenden waren bei allen Erprobungen in den Klassen anwesend. Dies wurde von den Lehrpersonen teils positiv bewertet, z. B. konnte der Aufsichtspflicht während der Gruppenarbeitsphasen besser gerecht werden. Eher negativ beurteilt wurde der zusätzliche organisatorische Aufwand, wie z. B. aufwändige Terminabsprachen mit allen Beteiligten. Keine der Lehrpersonen hatte zur Zeit des Interviews Mehrsprachiges Lesetheater ohne die Anwesenheit und ohne den Input der Forschenden in der Vorbereitungsphase angewendet. Es ist davon auszugehen, dass eine solche Erfahrung die Sicht der Lehrpersonen auf das Unterrichtsdesign prägt, in welche Richtung ist dabei nicht absehbar.

Es ist eine Stärke des Entwicklungs- und Forschungsprojekts, dass die Lesefördermethode Mehrsprachiges Lesetheater in enger Zusammenarbeit mit den Kooperationslehrkräften entwickelt und erprobt wurde. Allerdings bewerten die Lehrpersonen in den Interviews also ein Produkt, das sie selbst mitentwickelt haben. In den Gesprächen wurden die Lehrpersonen mehrfach darauf hingewiesen, dass das "fertig“ entwickelte Design im Fokus des Interviews steht. Inwieweit dies für die Lehrkräfte allerdings klar trennbar von früheren Versionen war, ist fraglich. Interessant wäre daher aus forschungs- und entwicklungstheoretischer Sicht, die Akzeptanz von neuen, MELT-unerfahrenen Lehrpersonen zu überprüfen.

Wir sehen Design-Based Research und eine auf Wirksamkeitsüberprüfung ausgerichtete Interventionsforschung nicht als konkurrierende, sondern als sich ergänzende Forschungsstrategien (vgl. Nieveen et al. 2006). Aufgrund der hier geschilderten Ergebnisse der Akzeptanzstudie betrachten wir MELT als ein Unterrichtsdesign, das von Lehrpersonen in der Praxis als praktikabel und nutzbringend angesehen wird. Ein nächster Schritt ist nun, die Wirksamkeit von Mehrsprachigem Lesetheater als Fördermaßnahme zu untersuchen. 